\title{
Correction to: The performance quality of LR, SVM, and RF for earthquake-induced landslides susceptibility mapping incorporating remote sensing imagery
}

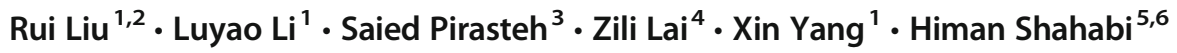 \\ Published online: 24 February 2021 \\ (C) Saudi Society for Geosciences 2021
}

\section{Correction to: Arabian Journal of Geosciences (2021) 14:259 https://doi.org/10.1007/s12517-021-06573-x}

The original version of this article unfortunately contained an error in the affiliation of one of the co-authors. The corresponding author missed the affiliation of the sixth co-author, Himan Shahabi, and was incorrectly presented as "Southwest Jiaotong University". The correct sixth co-author affiliation is "Department of Geomorphology, Faculty of Natural
Resources, University of Kurdistan, Sanandaj, Iran and Department of Zrebar Lake Environmental Research, Kurdistan Studies Institute, University of Kurdistan, Sanandaj, Iran. Given in this article is the corrected affiliation of sixth co-author.

The original article has been corrected.
The online version of the original article can be found at https://doi.org/ 10.1007/s12517-021-06573-x

Saied Pirasteh

sapirasteh@swjtu.edu.cn

Rui Liu

1r@cdut.edu.cn

Luyao Li

leeleoo@163.com

Zili Lai

1049962421@qq.com

Xin Yang

kkshiney@outlook.com

Himan Shahabi

h.shahabi@uok.ac.ir
College of Geophysics, Chengdu University of Technology, Sichuan 610059, China

2 Key Lab of Earth Exploration and Information Techniques of Ministry Education, Chengdu University of Technology, Sichuan 610059, China

3 Department of Surveying and Geoinformatics, Faculty of Geosciences and Environmental Engineering, Southwest Jiaotong University, The Western Park of the Hi-Tech Industrial Development Zone, Chengdu 611756, Sichuan, China

4 Dongpo District, Meishan, Sichuan Province, Housing and Urban-Rural Construction Bureau, Meishan 620000, Sichuan, China

5 Department of Geomorphology, Faculty of Natural Resources, University of Kurdistan, Sanandaj, Iran

6 Department of Zrebar Lake Environmental Research, Kurdistan Studies Institute, University of Kurdistan, Sanandaj, Iran 